\title{
Evidence for the production of type I collagen by adult rat hepatocytes in primary culture: immunohistochemical observations $^{1}$
}

\author{
HIROSHI KONOMI, RYU-ICHIRO HATA, JUNJIRO SANO, HIRONOBU SUNADA and \\ YUTAKA NAGAI \\ Department of Tissue Physiology, Medical Research Institute, Tokyo Medical and Dental University, Kanda- \\ surugadai, Chiyodaku, Tokyo 101, Japan
}

\begin{abstract}
The production of type I collagen by adult rat hepatocytes in primary culture was confirmed immunohistochemically, using antibodies to rat albumin and type I collagen.

The isolated hepatocytes formed a hepatic cord-like structure, when cultured for 2 days, and showed positive reaction with anti-albumin antibody, but not with antitype I collagen antibody. After 8 days of culture, however, the cells turned to show positive reactions with anti-type I collagen antibody as well as anti-albumin antibody. The results indicate that hepatocytes have acquired an ability to produce type I collagen during a period of 8 days in primary culture (cell differentiation), preserving the capability for albumin production.
\end{abstract}

Collagen is the major component of connective tissue. Although the synthesis of collagen has been studied primarily in cells originated from mesenchyme, its production has been demonstrated in a variety of non-mesenchymal cell systems (1) and recent studies have revealed that collagen is a family of proteins composed of at least five genetically distinct molecules (1).

Normal human liver contains relatively equal amounts of type I, III and IV (basement membrane) collagens. In cirrhotic livers, however, the anount of collagen of all these types increases and type I collagen becomes predominant at ihe later stage of the disease (9). Recently, the production of interstitial types of collagen and glycosaminoglycan by established cell lines originated from rat hepatocytes was reported

\footnotetext{
1 This is paper 7 on a series on the molecular mechanisms of hepatic fibrosis.

Abbreviations: Supplemented Williams' Medium E, Williams' medium E supplemented with $10^{-6} \mathrm{M}$ insulin, $10^{-5} \mathrm{M}$ dexamethasone, penicillin $(50 \mathrm{mg} / \mathrm{l})$ and with Fungizone $(0.25 \mathrm{mg} / \mathrm{l})$; Tris-buffered saline, $0.05 \mathrm{M}$ Tris- $\mathrm{HCl}$ buffer containing $0.11 \mathrm{M} \mathrm{NaCl}$
}

$(4,7,10)$. We could also demonstrate biochemically that even adult rat hepatocytes began to produce type I collagen during an 8-day-period of primary culture, although little collagen was produced by the cells in an early stage (second day) of culture (2).

To confirm whether cells responsible for collagen production are albumin-producing hepatocytes or non-hepatocyte cells which might be contaminated in the isolated hepatocyte fractions, we investigated albumin- and type I collagen-producing cells immunohistochemically using a cultured hepatocyte system.

Hepatocytes were isolated from adult male rats, Wistar strain, by the collagenase perfusion method followed by low speed centrifugation $(3,8)$. The cells were inoculated onto slide glass plates which were placed in $150 \mathrm{~mm}$ Falcon plastic culture dishes with Supplemented Williams' Medium E containing $10 \%$ fetal calf serum (2). Fibroblasts isolated from the abdominal skin of the same rat by treatment with $0.05 \%$ bacterial collagenase (Type I, Sigma Chemical Co., St. Louis, MO, U.S.A.)-0.1\% trypsin (DIFCO Laboratories, Detroit, MI, 
U.S.A.), were plated as described above. On the second and 8 th days of primary culture, the slide glass plates, on which the cells were cultured, were taken out, rinsed with several changes of $0.11 \mathrm{M} \mathrm{NaCl}$ in $0.05 \mathrm{M}$ Tris- $\mathrm{HCl}$ buffer, $\mathrm{pH} 7.4$ (Tris-buffered saline), and stored in $100 \%$ alcohol at $-70^{\circ} \mathrm{C}$ until used.

Rabbit antibody to rat type I collagen was purified by affinity chromatography using Sepharose 4B gels coupled with type I, II or III collagen. Anti-serum to type I collagen was successively passed over columns of type II and III collagens and finally adsorbed to and eluted from an antigen (type I collagen) column, as reported previously (5). The anti-type I collagen antibody thus prepared showed a reciprocal titer of $1 / 512$, and no cross-reaction with other types of collagen and albumin by the passive hemagglutination and hemagglutination inhibition assays. Rabbit antibody against rat serum albumin (Cappel Laboratories, PA, U.S.A.) was purified by affinity chromatography using $\mathrm{CH}$ Sepharose coupled with rat serum albumin after passing through a column of $\mathrm{CH}-$ Sepharose coupled with fetal calf serum used for the cell culture.

Sample cell specimens, pretreated with acetone for $10 \mathrm{~min}$, were incubated with anti-albumin antibody in a moist chamber for $60 \mathrm{~min}$ at room temperature, rinsed in several changes of Trisbuffered saline, then incubated with fluoresceinisothiocyanate-labeled anti-rabbit IgG goat IgG (MBL Co., Nagoya), diluted 1: 16 with the buffer, for $60 \mathrm{~min}$. After rinse in the buffer, the speci- mens were incubated with anti-type I collagen antibody for $60 \mathrm{~min}$, and then with biotinylatedanti-rabbit IgG goat IgG (Vector Laboratories, CA, U.S.A.), diluted 1:16 with the buffer, for $60 \mathrm{~min}$, as described above, and finally incubated with Rhodamine 600-avidin D (Vector Laboratories, CA, U.S.A.), diluted 1: 16 with the buffer, for $30 \mathrm{~min}$, then mounted in an $80 \%$ glycerol in the same buffer. The stained samples were observed with a Nikon FL fluorescence microscope. As a control, another set of cultured cells on slide glasses was incubated with rabbit IgG (absorbance at $280 \mathrm{~nm}=0.5$ ) immunized with Freund's complete adjuvant alone. We also stained rat and human fibroblasts, and rat hepatocytes co-cultured with rat fibroblasts to examine the specificity of the antibodies for inmunofluorescent stainings. Rat fibroblasts were stained only with anti-type I collagen antibody (Table 1). Hepatocytes and fibroblasts co-cultured for 2 days were stained with antialbumin and anti-type I collagen antibodies, respectively. However, human fibroblasts were stained neither with anti-rat albumin nor with anti-rat type I collagen antibodies. These results indicate that the antibodies to albumin and collagen used in this study are highly specific and the collagen produced by the fibroblasts does not deposit on the hepatocytes co-cultured. The sample cell specimens, which had been stained with antibodies to ablumin and type I collagen and examined by fluorescence microscopy, were subjected to hematoxylin-eosin staining and examined by light microscopy as reported pre-

Table 1 Frequency of the Appearance of Albumin- and/or Type I Collagen-Producing Cells during Primary Culture of Rat Hepatocytes or Dermal Fibroblasts

\begin{tabular}{lccc}
\hline \multicolumn{1}{c}{ Product } & \multicolumn{3}{c}{ Frequency } \\
& 2nd day & 8 th day $^{1}$ & $\begin{array}{c}\text { Dermal } \\
\text { fibroblast } \\
\text { 2nd day } 2\end{array}$ \\
\hline Albumin & $132 / 135$ & $74 / 83$ & nd \\
Type I collagen & nd & $64 / 83$ & $79 / 85$ \\
\hline
\end{tabular}

Observed by indirect immunofluorescent double stainings, using antibodies to rat albumin and type I collagen. Total cell numbers to be examined were counted after hematoxylin-eosin staining (denominator) and numbers of cells producing albumin and type I collagen were estimated from the cells showing positive reactions with corresponding antibodies (numerator).

1 The number of cells producing both type I collagen and albumin was 61 .

${ }^{2}$ Albumin- and type I collagen-producing cells were surveyed with different cultured dishes.

nd: not detected 
viously (6).

The isolated hepatocytes formed a hepatic cord-like structure, when cultured for 2 days, and showed positive reaction with anti-albumin antibody, but not with anti-type I collagen antibody (Fig. 1, a, b and c). After 8 days of culture, however, the cells turned to show positive reactions with anti-type I collagen antibody as well as anti-albumin antibody (Fig. 1, d, e and f), although the cell numbers were decreased and various sizes of polygonal cells lied scattered. When frequencies of albumin-producing and type I collagen-producing cells were examined in day-2 and day-8 cells, using the photos of immunofluorescent double stainings and hematoxylin-eosin stainings (Fig. 1), $89 \%$ of the cells remaining after 8 days of culture was albuminproducing and $77 \%$ was type I collagen-producing (Table 1). The cells simultaneously producing albumin and type I collagen were $73 \%$ of total cells counted, indicating that the collagen was produced by hepatocytes in this culture system, but not by non-albumin-producing cells which might have been present in the isolated cell preparation and rapidly grown during the culture. Day-2-cells showed that $98 \%$ of the cells was albumin-producing with no type I collagen production (Table 1).

Analysis of the collagen production during primary culture of hepatocytes has revealed that total collagen synthesis, in which type I collagen is predominant, increased 7 to 9 times on the 8th day, regardless of the addition of aphidicolin, an inhibitor of DNA polymerase $\alpha$, or not (2). Moreover, albumin-production per cell increased after 8 days of culture (unpublished data).

The results described above provide a strong line of evidence for the production of interstitial collagen by hepatocytes. The cells have acquired an ability to produce collagen under certain conditions such as primary culture (cell differentiation). This suggests that the hepatocyte may participate in liver connective tissue metabolism under certain pathological conditions. Studies along this line are now in progress.

We wish to thank Dr Yutaka Tsukada for his useful advices on the purification of rat serum albumin. This work was supported in part by grants from the
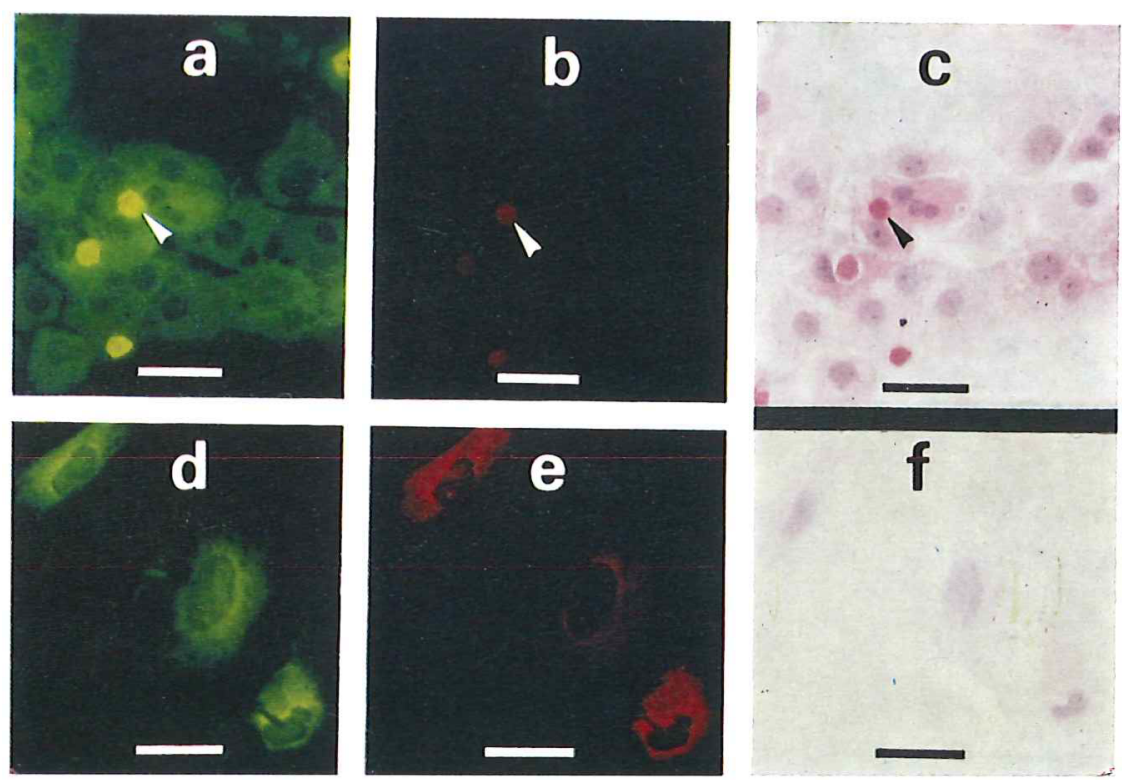

Fig. 1 Indirect immunofluorescent double stainings of cultured rat hepatocytes with antibodies to rat albumin and type I collagen. Fluoresceinisothiocyanate (FITC) and rhodamine were used for the staining of albumin (a,d) and type I collagen (b, e), respectively. The horizontal bars represent $50 \mu \mathrm{m}$. The cells on day-2 (a, b, c) and day-8 (d, e, f) of primary culture were first stained with antibody to albumin and then with that to type I collagen. The arrowhead indicates the identical point in $\mathrm{a}, \mathrm{b}$ and $\mathrm{c}$, which is the nucleus of dead cell showing strong autofluorescence. After the double staining, the same cell specimen was stained with hematoxylin-eosin (c, f). 
Ministry of Education, Science and Culture (55703 and Special Project Research on Connective Tissue Diseases), from the Intractable Diseases Division, Public Health Bureau, the Ministry of Health and Welfare, Japan (Project Team for the Research on Connective Tissue Disorder Research Committee) and from the Yamanouchi Foundation for Research on Metabolic Disorders.

Received for publication 10 April 1982; and in revised form 8 May 1982

\section{REFERENCES}

1. Bornstein P. and SAge H. (1980) Structurally distinct collagen types. Annu. Rev. Biochem. 49, 957-1003

2. Hata R. and Nagai Y. (1980) Differentiation of hepatocytes into type I collagen producing cells during primary culture in the presence of aphidicolin (inhibitor of DNA polymerase $\alpha$ ). Biochem. Int. 1, 567-573

3. Hata R., NinomiYa Y., NAGai Y, and Tsukada Y. (1979) Collagen production by rat liver parenchymal cells in primary culture. Proc. $\mathrm{Ja}$ pan Acad. Ser. B. 55, 531-536

4. Hata R., NinomiYa Y., Nagai Y. and Tsukada Y. (1980) Biosynthesis of interstitial types of collagen by albumin-producing rat liver parenchy- mal cell (hepatocyte) clones in culture. Biochemistry 19, 169-176

5. Konomi H., Hori H., Sano J., Sunada H., Hata R., Fujiwara S. and Nagai Y. (1981) Immunohistochemical localization of type I, II, III and IV collagens in the lung. Acta Pathol. Japon. 31, 601-610

6. Konomi H., Sano J. and Nagal Y. (1981) Immunohistochemical localization of types I, III and IV (basement membrane) collagens in the lymph node: Co-distribution of types I and III collagens in the reticular fibers. Biomedical Res, 2, 536-545

7. Ninomiya Y., Hata R. and Nagai Y. (1980) Glycosaminoglycan synthesis by liver parenchymal cell clones in culture and its change with transformation. Biochim. Biopliys. Acta 629, 349-358

8. Ninomiya Y., Hata R. and Nagai, Y. (1981) Active synthesis of glycosaminoglycans by liver parenchymal cells in primary culture. Biochim. Biophys. Acta 675, 248-255

9. Rojkind M., Giambrone M. and Biempica L. (1979) Collagen types in normal and cirrhotic liver. Gastroenterology 76, 710-719

10. Sakakibara K., Saito M., Umeda M., Enaka K. and TsuKaDA Y. (1976) Native collagen formation by liver parenchymal cells in culture. Nature 262, 316-318 